\title{
Panel Regression of Arbitrarily Distributed Responses
}

\author{
Gordon G. Bechtel \\ University of Florida and Florida Research Institute
}

\begin{abstract}
The primary advantage of panel over cross-sectional regression stems from its control for the effects of omitted variables or "unobserved heterogeneity". However, panel regression is based on the strong assumptions that measurement errors are independently identically (i.i.d.) and normal. These assumptions are evaded by design-based regression, which dispenses with measurement errors altogether by regarding the response as a fixed real number.

The present paper establishes a middle ground between these extreme interpretations of longitudinal data. The individual is now represented as a panel of responses containing dependently non-identically distributed (d.n.d) measurement errors. Modeling the expectations of these responses preserves the Neyman randomization theory, rendering panel regression slopes approximately unbiased and normal in the presence of arbitrarily distributed measurement error. The generality of this reinterpretation is illustrated with German Socio-Economic Panel (GSOEP) responses that are discretely distributed on a 3-point scale.
\end{abstract}

Key words: Longitudinal weights, panel deviations, population of panels, single-stage panel sampling, stochastic measurement error, survey response expectation.

\section{Random Individual-Wave Variables}

Design-based sampling postulates the respondent in a fixed observable state that $\mathrm{s}(\mathrm{he})$ reports as a discrete rating, such as 012 , or recalls on a continuous monetary scale. Thus, the value recorded on an opinion poll or economic survey is regarded as a real number in waiting. More realistically, however, the survey response may be viewed as a random variable containing measurement error (cf. Diggle, Liang, and Zeger, 1994). The present paper favors this more plausible interpretation and extends Bechtel's (2007) treatment of cross-sectional regressions to longitudinal regressions involving repeated measurements. These measurements make up a panel of random variables, which may be dependently non-identically distributed (d.n.d) within each respondent. A finite population of 
these panels then gives rise to a finite population of realized random individualwave variables. Each realization is a momentary numerical value governed by an individual-wave-specific mean and variance. This approach retains and enhances design-based regression, whose slopes are still normally distributed (over samples) for any stochastic distributions (over realizations) that prevail for individualwave-specific responses.

Section 2 describes an unbalanced longitudinal population along with a singlestage sample of panels. Section 3 regresses response expectations over this population, defining new target parameters as functions of these expectations. Using the Horvitz-Thompson theorem, Sections 4, 5, and 6 show that these new parameters are estimated by the well-known design-based coefficients. Section 7 describes a user-friendly computation of these coefficients with STATA software. Section 8 uses this software to evaluate predictors of environmental concern in the German Socio-Economic Panel. The final section summarizes distributionfree panel regression and reemphasizes its applicability to arbitrarily distributed survey responses.

\section{The Population and Sample of Panels}

The term panel is used here to denote an intra-individual sequence of wave measures $Y_{i t}$. This sequence is illustrated by a single row in Table 1, where $t=1$ for individual $i$ 's first appearance. A population of panels is a finite set of panels exemplified by the seven rows in Table 1. This population is "unbalanced" because different individuals make different numbers of wave appearances. An unbalanced population of panels is also a series of incomplete censuses, such as the four columns in Table 1.

Table 1: An unbalanced longitudinal population of panels

\begin{tabular}{lcccc}
\hline Panel & Wave 1 & Wave 2 & Wave 3 & Wave 4 \\
\hline Individual 1 & $\mathbf{Y}_{11}$ & $\mathbf{Y}_{12}$ & $\mathbf{Y}_{13}$ & $\mathbf{Y}_{14}$ \\
Individual 2 & $Y_{21}$ & $Y_{22}$ & $Y_{23}$ & \\
Individual 3 & $\mathbf{Y}_{31}$ & $\mathbf{Y}_{32}$ & $\mathbf{Y}_{33}$ & \\
Individual 4 & $Y_{41}$ & $Y_{42}$ & & \\
Individual 5 & $\mathbf{Y}_{51}$ & & & \\
Individual 6 & $Y_{61}$ & $Y_{62}$ & & \\
Individual 7 & $Y_{71}$ & $Y_{72}$ & $Y_{73}$ & \\
\hline
\end{tabular}

The boldface rows in Table 1 exhibit an unbalanced sample of three panels drawn without replacement from our population of seven panels. Because every wave appearance in each sampled panel is measured, Table 1 illustrates singlestage cluster sampling (Lohr, 1999, pp. 136-145), which is called single-stage 
panel sampling in the sequel. In this example a sample of eight individual-wave measures are drawn from a population of eighteen individual-wave values by single stage panel sampling.

In the sequel $Y_{i t}$ in Table 1 plays three roles: a realizable random variable, its realized value which is a real number, and an observed (i.e. sampled) realized value. A panel can be viewed, therefore, as a cluster of random variables or as a cluster of fixed realizations. The following sections emphasize the importance of stochastic measurement error in these distinctions.

\section{Regressing Expectations in the Population of Panels}

\subsection{Stochastic measurement error}

The present paper uses survey data that sharply departs from the (usually assumed) continuity, normality, and homoscedasticity of the panel response variable (cf. Baltagi, 2001; Hsiao, 2003). Here $Y_{i t}=0,1,2$ denotes a rating of environmental concern by German panelist $i$ on wave $t$. The response options and coding for the GSOEP's three-point scale are:

Not concerned at all (0) Somewhat concerned (1) Very concerned (2)

This score is a discrete random variable that may be decomposed as

$$
\begin{aligned}
Y_{i t} & =H_{i t}+E_{i t} \\
& =\alpha_{i}^{*}+\beta_{1}^{*} X_{1 i t}+\cdots+\beta_{k}^{*} X_{k i t}+\gamma_{i t}^{*}+E_{i t},
\end{aligned}
$$

where

$$
E\left(Y_{i t}\right)=H_{i t}=\alpha_{i}^{*}+\beta_{1}^{*} X_{1 i t}+\cdots+\beta_{k}^{*} X_{k i t}+\gamma_{i t}^{*},
$$

$\alpha_{i}^{*}$ is a fixed individual intercept, $X_{1 i t}, \ldots, X_{k i t}$ are fixed individual-wave-specific predictors, $\gamma_{i t}^{*}$ is a fixed individual-wave effect on $H_{i t}$, and $E_{i t}=Y_{i t}-H_{i t}$ is a measurement error for individual $i$ on wave $t$, with $E\left(E_{i t}\right)=0$ and $\operatorname{Var}\left(E_{i t}\right)=\sigma_{i t}^{2}$.

In (3.1) our unit of interest, individual $i$ on wave $t$, is represented by a pair of parameters; namely, a mean $i t$ and variance $\sigma_{i t}^{2}$ that determine an idiosyncratic, wave specific probability distribution on the scale 012 . The mean $H_{i t}$ is continuous in the interval $[0,2]$ and is composed of an individual intercept, individual-wave-specific predictors, and an effect that is unique to individual $i$ on wave $t$. This latter effect $\gamma_{i t}^{*}$ saturates the linear model for the $H_{i t}$ in (3.1), i.e. the structure

$$
\alpha_{i}^{*}+\beta_{1}^{*} X_{1 i t}+\cdots+\beta_{k}^{*} X_{k i t}+\gamma_{i t}^{*}
$$

fits the $H_{i t}$ exactly without constraining these expectations. 
The $E_{i t}$ in (3.1) may be dependently non-identically distributed (d.n.d.) over waves within individuals. Fixing individual $\mathrm{i}$ and wave $\mathrm{t}$, the random variable $E_{i t}$ can be displayed as follows:

$$
\begin{array}{ccc}
p_{0 i t} & p_{1 i t} & p_{2 i t} \\
0-H_{i t} & 1-H_{i t} & 2-H_{i t}
\end{array}
$$

The (unknown) response probabilities $p_{0 i t}, p_{1 i t}$, and $p_{2 i t}$ for not concerned at all, somewhat concerned, and very concerned are arbitrarily distributed over the points $0-H_{i t}, 1-H_{i t}$, and $2-H_{i t}$. The standard deviation $\sigma_{i t}$ on this 3-point error scale denotes uncertainty in $i$ 's rating of environmental concern. A small $\sigma_{i t}$ represents a precisely reporting individual with a narrow error distribution. A broad error distribution has a large $\sigma_{i t}$ characterizing an individual with less consistent ratings over repeated realizations of the random variable $Y_{i t}$.

\subsection{New target parameters for design-based regression}

In equation (3.1) the intercept $\alpha_{i}^{*}$, the slopes $\beta_{1}^{*}, \ldots, \beta_{k}^{*}$, and the effects $\gamma_{i t}^{*}$ will be uniquely identified by the ordinary-least-squares (OLS) condition that $\sum_{i t} \gamma_{i t}^{* 2}$ is minimal when the population of expectations $H_{i t}$ is regressed on the population of predictors $X_{1 i t}, \ldots, X_{k i t}$. This OLS identification of $\boldsymbol{\beta}^{*}=\left(\beta_{1}^{*}, \ldots, \beta_{k}^{*}\right)^{T}$ is given by the following function of these expectations:

$$
\boldsymbol{\beta}=\left[\sum_{i t} x_{i t} x_{i t}^{T}\right]^{-1} \sum_{i t} x_{i t} \eta_{i t}
$$

for $i=1, \ldots, N ; t=1, \ldots, T_{i}$, where $x_{i t}=X_{i t}-X_{i .}, \eta_{i t}=H_{i t} .-H_{i}$.

In (3.2) $X_{i t}=\left(X_{1 i t}, \ldots, X_{k i t}\right)$, and $X_{i \ldots}$ and $H_{i}$. are the means of $X_{i t}$ and $H_{i t}$ within panel $i$ (StataCorp. 2001, p. 437 ; Hsiao 2003, pp. 30-33). Thus $\boldsymbol{\beta}$ in (3.2) is expressed in terms of the deviations of response expectations and predictors from their panel means. Equation (3.2) selects the unique parameterization $\alpha_{i}$, $\beta_{1}, \ldots, \beta_{k}, \gamma_{i t}$ from an infinite set $\left\{\alpha_{i}^{*}, \beta_{1}^{*}, \ldots, \beta_{k}^{*}, \gamma_{i t}^{*}\right\}$ of exact characterizations of the $H_{i t}$. This defines the new target parameters of design-based regression as $\beta_{1}, \ldots, \beta_{k}$.

\section{Sampling from a Realized Population of Panels}

\subsection{Single-stage panel sampling}

Our clustered population of individuals, each containing $T_{i}$ survey waves for $i=1, \ldots, N$, is anchored by $\sum_{i} T_{i}$ expectations $H_{i t}$. In Table 1 , for example, $i=$ $1, \ldots, 7$ panels and $\sum_{i} T_{i}=18$ individual-waves. Now let the random variable $Y_{i t}$ be realized for every individual-wave in the population of panels. This population 
realization occurs in a hypothetical (but possible) series of incomplete censuses. A single-stage cluster sample of $n$ panels is then drawn without replacement from this population of $N$ panels. The sample consists of $\sum_{i} T_{i}$ ratings $Y_{i t}$ for $i=$ $1, \ldots, n$. In Table $1 \sum_{i} T_{i}=8$ individual-waves are drawn from $i=1,2,3$ sampled panels. This setup reinterprets conventional design-based sampling which treats the $Y_{i t}$ as constants rather than realizations of random variables.

\subsection{Longitudinal weights}

The sample inclusion probability for a panel is the cross-sectional inclusion probability of its initial wave multiplied by the retention probabilities for its subsequent waves. These retention probabilities are "the conditional probabilities of remaining in the panel" over these remaining waves (Haisken-DeNew and Frick 2005 , p. 171). For example, the sample inclusion probability $\pi_{3}$ for individual 3 in Table 1 is her (his) cross-sectional inclusion probability in wave 2 multiplied by her (his) retention probabilities for waves 3 and 4 . The sample inclusion probability $\pi_{5}$ for individual 5 , however, is simply her (his) cross-sectional inclusion probability in wave 2 . The final longitudinal weights for individuals 3 and 5 are the reciprocals of their inclusion probabilities, i.e. $w_{3}=1 / \pi_{3}$ and $w_{5}=1 / \pi_{5}$.

In the German Socio-Economic Panel each respondent is assigned a crosssectional weight and a longitudinal weight for each wave. The cross-sectional weight for panel $i$ 's first participating year is multiplied by the longitudinal weights for her (his) subsequent participating years. Each longidudinal weight is the reciprocal of $i$ 's "staying" probability for that subsequent year, i.e. the conditional probability $\mathrm{s}(\mathrm{he})$ participates in that wave and in the previous waves of her (his) panel (Haisken-DeNew and Frick 2005, p. 180). The product of panel $i$ 's initial cross-sectional weight and subsequent longitudinal weights produces $i$ 's final longitudinal weight $w_{i}$. This weight $w_{i}$ covers the sequence of years individual i is monitored within the time span 1999-2005.

\section{Estimating Panel Regression Coefficients}

\subsection{The conventional moving target}

Each element of the $k \times k$ matrix $\sum_{i t} x_{i t} x_{i t}^{T}$ is a population sum of products, as is each element of the $k \times 1$ vector $\sum_{i t} x_{i t} y_{i t}$ (Lohr, 1999, p. 360). Each sum of products contains panel deviation scores

$$
\begin{aligned}
x_{i t} & =X_{i t}-X_{i} . \\
y_{i t} & =Y_{i t}-Y_{i} .
\end{aligned}
$$

for $t=1, \ldots, T_{i}$, where $X_{i}$. and $Y_{i}$. are the means of $X_{i t}$ and $Y_{i t}$ within panel 
$i$ for $i=1, \ldots, N$. Due to Horvitz and Thompson (1952), unbiased estimates of the matrix $\sum_{i t} x_{i t} x_{i t}^{T}$ and the vector $\sum_{i t} x_{i t} y_{i t}$ are given by $\sum_{i t} w_{i} x_{i t} x_{i t}^{T}$ and $\sum_{i t} w_{i} x_{i t} y_{i t}$ for $i=1, \ldots, n ; t=1, \ldots, T_{i}$. The weight $w_{i}$ is individual $i$ 's final longitudinal weight described in Section 4.2. When the sample size $n$ is large, the Horvitz-Thompson (HT) estimator

$$
\mathbf{B}=\left[\sum_{i t} w_{i} x_{i t} x_{i t}^{T}\right]^{-1} \sum_{i t} w_{i} x_{i t} y_{i t}
$$

for $i=1, \ldots, n ; t=1, \ldots, T_{i}$, is consistent and almost unbiased for the conventional target parameter

$$
\boldsymbol{\theta}=\left[\sum_{i t} x_{i t} x_{i t}^{T}\right]^{-1} \sum_{i t} x_{i t} y_{i t}
$$

for $i=1, \ldots, N ; t=1, \ldots, T_{i}$.

The unbiasedness of $\mathbf{B}$ is approximate because it is the product of matrix and vector estimators (Binder, 1983; Nathan, 1988, pp. 255-256; Thompson, 1997, pp. 106-107; Valliant, Dorfman, and Royall, 1999, pp. 40-41; Lohr, 1999, pp. 354-361; StataCorp. 2001, Volume 4, pp. 29-30; Chaudhuri and Stenger 2005, pp. 264-265.) The parameter $\boldsymbol{\theta}$ in (5.2) is called a moving target because it is a function of the transient deviations $y_{i t}=Y_{i t}-Y_{i}$.

\subsection{The new stationary target}

The important result here is that the conventional formula (5.1) also estimates the more profound and anchored target parameter (3.2), which is a function of constant expectations $H_{i t}$ rather than momentary realizations $Y_{i t}$. To obtain this result we take the expected value of (5.2) over realizations of the stochastic ratings $Y_{i t}$ :

$$
\begin{aligned}
E(\theta) & =\left[\sum_{i t} x_{i t} x_{i t}^{T}\right]^{-1} \sum_{i t} x_{i t} E\left(y_{i t}\right) \\
& =\left[\sum_{i t} x_{i t} x_{i t}^{T}\right]^{-1} \sum_{i t} x_{i t} \eta_{i t}=\boldsymbol{\beta}
\end{aligned}
$$

for $i=1, \ldots, N ; t=1, \ldots, T_{i}$. Because $E\left(\theta_{j}\right)=\beta_{j}$ and $\operatorname{Var}\left(\theta_{j}\right) \rightarrow 0$ as the number of panels $N \rightarrow \infty$, the differences $\theta_{j}-\beta_{j}$ for $j=1, \ldots, k$ are infinitesimal for a given large population realization. Thus $\mathbf{B}$ in (5.1), which is almost unbiased for $\boldsymbol{\theta}$ in (5.2), is almost unbiased for $\boldsymbol{\beta}$ in (3.2) as well. 


\section{Normality and Variances of the Estimated Coefficients}

Fixing the momentary population realizations $Y_{i t}$ for $i=1, \ldots, N$ and $t=$ $1, \ldots, T_{i}$, the resulting reals $\theta_{1}, \ldots, \theta_{k}$ in (5.2) become the classic target parameters of design-based regression. Therefore, a strict design-based argument using the $\theta_{j}$ can be given for the normality over large samples of each element $B_{j}$ in $\mathbf{B}$. This provides a statistic for testing hypotheses about the new target parameter $\beta_{j}$ against the conventional estimate $B_{j}$.

First, given the population realizations $Y_{i t}$, the coefficient $\theta_{j}$ for $j=1, \ldots, k$ can be written as a smooth function of cross-product totals in the population

$$
\left\{y_{i t}, x_{1 i t}, \ldots, x_{k i t}: i=1, \ldots, N ; t=1, \ldots, T_{i}\right\}
$$

of deviation scores. Then, from the sample

$$
\left\{y_{i t}, x_{1 i t}, \ldots, x_{k i t}: i=1, \ldots, n ; t=1, \ldots, T_{i}\right\}
$$

the estimate $B_{j}$ can be written as the same function of HT estimators of these population totals. The HT estimators are corresponding sample totals of cross products with each term weighted by $w_{i}$. For example, the sample total

$$
\sum_{i t} w_{i} x_{1 i t} y_{i t} \quad \text { for } i=1, \ldots, n ; t=1, \ldots, T_{i}
$$

is an HT estimator of the population total $\sum_{i t} x_{1 i t} y_{i t}$ for $i=1, \ldots, N ; t=$ $1, \ldots, T_{i}$ (cf. Lohr, 1999, pp. 352-360; Thompson, 1997, pp. 106-108).

The asymptotic normality of HT estimators (Sen, 1988, pp. 313-328) may now be used to justify the asymptotic normality of $B_{j}$, which is a nonlinear function of these estimators. A "linearization" of the error $B_{j}-\theta_{j}$ is provided by the first-order approximation $B_{j}-\theta_{j} \approx \epsilon_{j}$, where $\epsilon_{j}$ is the linear term in a Taylor series expansion of this error. Asymptotic multivariate normality of the HT estimators then implies that $\left(B_{j}-\theta_{j}\right) / \sqrt{\operatorname{Var}\left(\epsilon_{j}\right)}$ is asymptotically $N(0,1)$ (Lehmann, 1999, pp. 253-269, 309-315; Lohr, 1999, pp. 290-293, 310, 352-360; Thompson, 1997, pp. 58-64, 106-111). The estimate $\operatorname{Var}\left(\epsilon_{j}\right)$ of $\operatorname{Var}\left(\epsilon_{j}\right)$ is given by the $j$-th diagonal element of the matrix $\operatorname{Var}(\mathbf{B})$ in (6.2) and is computed by software described in Section 7. Due to the infinitesimal difference between $\theta_{j}$ and $\beta_{j}$, the statistic

$$
t=\frac{B_{j}-\beta_{j 0}}{\sqrt{\operatorname{Var}\left(\epsilon_{j}\right)}}
$$

may be used to test an hypothesis $H: \beta_{j}=\beta_{j 0}$ about our target coefficient $\beta_{j}$. This test for $\beta_{j 0}=0$ is illustrated for the regression coefficients in Table 2 below. 
Table 2: Regression coefficients for predicting environmental concern $\left(R^{2}=.28\right)$

\begin{tabular}{lccc}
\hline Concern predictor & Coefficient & Standard error & $t$-statistic \\
\hline General economic development & .045 & .011 & 4.28 \\
Your health & .133 & .011 & 12.26 \\
Maintaining peace & .214 & .012 & 17.55 \\
Crime in Germany & .111 & .011 & 10.13 \\
Hostility toward foreigners & .072 & .010 & 6.88 \\
or minorities in Germany & & & \\
Age & -.007 & .002 & 2.93 \\
\hline
\end{tabular}

Finally, again using "linearization", an estimate of the entire covariance matrix of $\mathbf{B}$ is

$$
\operatorname{Var}(\mathbf{B})=\left(\sum_{i t} w_{i} x_{i t} x_{i t}^{T}\right)^{-1} \operatorname{Var}\left[\sum_{i t} w_{i} x_{i t}^{T}\left(y_{i t}-x_{i t}^{T} \mathbf{B}\right)\right]\left(\sum_{i t} w_{i} x_{i t} x_{i t}^{T}\right)^{-1},
$$

where $i=1, \ldots, n$ and $t=1, \ldots, T_{i}$ (Lohr, 1999, pp. 359-361; StataCorp., 2001, Volume 4, pp. 29-30). As described in Section 4.2, the longitudinal weight $w_{i}$ in (6.2) is fixed over the $T_{i}$ waves in individual $i$ 's panel. The $j$-th diagonal of $\operatorname{Var}(\mathbf{B})$ is the estimated variance of $B_{j}$ in the denominator of (6.1). Again note that the covariance estimator in (6.2) is expressed in panel deviation scores.

\section{Software}

The estimated regression coefficients, along with their standard errors and test statistics, are easily calculated with the STATA commands:

$$
\begin{aligned}
& \text { svyset pweight longitudinal_weight } \\
& \text { svyset psu panel } \\
& \text { svyreg devY devX1 ... devXk, noconstant }
\end{aligned}
$$

(StataCorp. 2001, Volume 4, pp. 18- 31). In (7.1) longitudinal_weight is the variable containing the longitidunal weights. In (7.2) panel is the variable containing the panel identifications. The definitions of the deviation variables in (7.3) are:

$$
\begin{aligned}
\operatorname{dev} Y= & Y_{i t}-Y_{i \cdot}=y_{i t} \\
\operatorname{dev} X_{1}= & X_{1 i t}-X_{1 i}=x_{1 i t} \\
& \cdots \cdots \\
\operatorname{dev} X_{k}= & X_{k i t}-X_{k i \cdot}=x_{k i t}
\end{aligned}
$$

The option noconstant in (7.3) suppresses the intercept because the response variable and its predictors are deviations from their panel means. 
For large samples these three STATA commands return (approximately) normal and unbiased estimates $B_{1}, \ldots, B_{k}$ in the presence any distributions of the measurement errors $E_{i t}$ in (3.1). The standard errors of the estimated coefficients reflect the effects of these measurement errors on coefficient variance.

\section{Environmental Concern in Germany}

\subsection{The GSOEP for 1999-2005}

Because Germany has been at the forefront of environmental protection, the present investigation of environmental concern relies upon the well-established German Socio-Economic Panel. The first wave of the GSOEP was carried out in 1984 in the Federal Republic of Germany. The panelists studied here are residents of the former Federal Republic living in private households whose head is not Turkish, Greek, Yugoslavian, Spanish, or Italian. These respondents are known as the "west German sample" of the GSOEP (Haisken-DeNew and Frick 2005, p. 19).

The GSOEP interviews are conducted face-to-face with all persons in a household aged 16 and over. Our west German sample consists of 6634 respondents measured within the seven years of the present study, i.e. 1999-2005. Further details on the English Language Public Use File of the GSOEP, including instructions for obtaining the data, have been given by Wagner, Burkhauser, and Behringer (1993).

The survey firm Infratest Burke Sozialforschung in Munich carries out the fieldwork for the GSOEP. In addition to demographic information, the GSOEP questionnaire contains "objective" measures such as income and unemployment, as well as "subjective" ratings of satisfactions, worries and fears of the German population.

\subsection{The GSOEP items for rating worry}

The GSOEP contains ratings of concern, or worry, about various living conditions in Germany, Europe, and the world. These items are prefaced with the question:

What is your attitude toward the following areas - are you concerned about them?

The areas of concern studied here are:

Environmental protection; General economic development; Your health;

Maintaining peace; Crime in Germany; Hostility toward foreigners or minorities in Germany

The response scale and coding for these items were described in Section 3.1 as: 
Not concerned at all (0) Somewhat concerned (1) Very concerned (2)

\subsection{The weighted panel regression}

Using the STATA commands (7.1), (7.2), and (7.3), equation (3.1) is estimated as

$$
\hat{Y}_{i t}=A_{i}+B_{1} X_{1 i t}+\cdots+B_{6} X_{6 i t} .
$$

Five significant predictors of environmental concern, along with age, are exhibited in Table 2. This west German regression was run over 34269 individual-wave measures generated by 6634 panels from 1999 to 2005. These panels ranged from one to seven waves, with an average of 5.2 waves. The estimates $A_{1}, \ldots, A_{6634}$ of the individual effects are not included in this report.

The five concern coefficients in Table 2 are commensurate because they share the three-point rating scale in Section 6.2. The strongest predictor of environmental concern is worry about maintaining peace, followed by worries about your health and crime in Germany. The negative age coefficient reveals that younger Germans have greater environmental concern.

The predictors in Table 2, except for your health, suggest that German environmental concern has an altruistic societal, rather than selfish individualistic, orientation. This is supported by the finding that potential regressors, such as personal dwelling satisfaction, and concern with your own economic situation, failed to reach statistical significance in predicting environmental concern.

\section{Summary}

An unbalanced longitudinal population of real numbers is reinterpreted as a set of momentary realizations of random variables $Y_{i t}$, each governed by the parameters $H_{i t}$ and $\sigma_{i t}^{2}$ for individual $i$ on wave $t$. (See Table 1.) This reinterpretation better justifies the usual design-based regression estimates and their standard errors. It opens up panel regression to design-based theory, response weighting, and arbitrary stochastic responding without reference to an abstract superpopulation (cf. Skinner, Holt, and Smith 1989).

The primary advantage of panel over cross-sectional regression lies in the possibility of bringing variable intercepts $\alpha_{i}^{*}$ into the model. These individual effects, which reside in the error term of a cross-sectional model, bias regression coefficients if they are related to both the response and its predictors. This potential bias is removed by (3.1) which contains $\alpha_{i}^{*}$ as an estimable effect. However, this individual effect, and the non-estimable individual-wave effect $\gamma_{i t}^{*}$ in (3.1), are not needed in defining our target $\boldsymbol{\beta}$ in (3.2) and its estimate $\mathbf{B}$ in (5.1).

The present panel extension of Bechtel (2007) differs from model-based sampling, where the finite population of realizations is itself a sample from a "su- 
perpopulation" with assumed distribution and covariance properties (cf. Binder 1983; Nathan 1988; Skinner, Holt, and Smith 1989; Thompson 1997; Valliant, Dorfman, and Royall 1999; Binder and Roberts 2003). Here this "superpopulation" is simply a finite set of arbitrarily distributed wave variables that are clustered by individuals. These random variables are realized as responses to a hypothetical (but possible) sequence of incomplete censuses. The targets of inference are population regression coefficients that are functions of the expectations of individual-wave realizations. This longitudinal population, and its limited target parameters, establish a plausible bridge between design- and model-based regression theory.

Finally, the estimate $\mathbf{B}$ in (5.1) of the target $\boldsymbol{\beta}$ in (3.2) is asymptotically normal and almost unbiased (over samples) whatever the distribution (over realizations) of $Y_{i t}$ in the panel population. Thus, the reinterpretation of $Y_{i t}$ as a stochastic response rather than a fixed real number is a step forward in the Neyman paradigm (Bellhouse, 1988). By allowing this response to be arbitrarily stochastic, formulas (3.2) and (5.1) also strip away the distribution assumptions thought to be necessary for panel regression (cf. Baltagi, 2001; Hsiao, 2003).

\section{Acknowledgements}

This work has been supported by the University of Florida's Warrington College of Business. The author thanks the JDS editor and referee whose responses to the first draft have greatly improved the paper. The German Socio-Economic Panel data were supplied to the author under a contract with the German Institute for Economic Research in Berlin. These data were obtained from the Cornell University English Language Public Use File of the GSOEP. None of the ideas or analyses in the present paper are attributable to the University of Florida, Cornell University, or the German Institute for Economic Research.

\section{References}

Baltagi, B. H. (2001). Econometric Analysis of Panel Data, 2nd edition. Wiley.

Bechtel, G. G. (2007). Distribution-free regression: Reinterpreting design-based sampling. Journal of Data Science 5, 535-553.

Bellhouse, D. R. (1988). A brief history of random sampling methods. In Handbook of Statistics, Volume 6 (Sampling) (Edited by P.R. Krishnaiah and C.R. Rao), pp. 1-14, North Holland.

Binder, D. A. (1983). On the variances of asymptotically normal estimators from complex samples. International Statistical Review 51, 279-292. 
Binder, D. A. and Roberts, G.R. (2003). Design-based and model-based methods for estimating model parameters. In Analysis of Survey Data (Edited by R. L. Chambers and C. J. Skinner), pp. 29-48, Wiley.

Chaudhuri, A. and Stenger, H. (2005). Survey Sampling: Theory and Methods, 2nd edition. Chapman and Hall.

Diggle, P. J., Liang K-Y and Zeger S. L. (1994). Analysis of Longitudinal Data. Oxford University Press.

Haisken-DeNew, J. P. and Frick, J. R. (2005). Desktop Companion to the German Socio- Economic Panel, Version 8.0 - December 2005. German Institute for Economic Research.

Hsiao, C. (2003). Analysis of Panel Data, 2nd edition. Cambridge University Press.

Horvitz, D. G. and Thompson, D. J. (1952). A generalization of sampling without replacement from a finite universe. Journal of the American Statistical Association 47, 663-685.

Lehmann, E. L. (1999). Elements of Large-Sample Theory. Springer.

Lohr, S. L. (1999). Sampling: Design and Analysis. Duxbury Press.

Nathan, G. (1988). Inference based on data from complex sample designs. In Handbook of Statistics, Volume 6 (Sampling) (Edited by P.R. Krishnaiah and C.R. Rao ), pp. 247-266, North Holland.

Sen, P. K. (1988). Asymptotics in finite population sampling. In Handbook of Statistics, Volume 6 (Sampling) (Edited by P.R. Krishnaiah and C.R. Rao), pp. 291-331, North Holland.

Skinner, C. J., Holt, D. and Smith, T. M. F. (1989). Analysis of Complex Surveys. Wiley.

StataCorp. (2001). Stata Statistical Software: Release 7.0. Stata Corporation.

Thompson, M. E. (1997). Theory of Sample Surveys. Chapman and Hall.

Valliant, R., Dorfman, A. H. and Royall, R. M. (1999). Finite Population Sampling and Inference: A Prediction Approach. Wiley.

Wagner, G. G., Burkhauser, R. V. and Behringer, F. (1993). New data bases: The English language public use file of the German Socio-Economic Panel. The Journal of Human Resources 28, 429-433.

Received August 20, 2007; accepted November 29, 2007.

Gordon G. Bechtel

University of Florida and Florida Research Institute

P.O. Box 117155

Gainesville, Florida 32611-7155, USA

bechtel@ufl.edu 